\title{
Cemented Total Knee Arthroplasty- A Short Term Clinical, Functional Assessment and Outcome Analysis
}

\author{
Meenakshi Sundaram Kandasamy리 Kandaswamy Ganesh Shankar Kandaswamy22, Sathish Kumar Rajaram³, \\ Vijayan Gopala Krishna Kurup ${ }^{4}$
}

${ }^{1}$ Department of Orthopaedics, VMKV Medical College and Hospital, Salem, Tamilnadu, India. ${ }^{2}$ Department of Orthopaedics, VMKV Medical College and Hospital, Salem, Tamilnadu, India. ${ }^{3}$ Department of Orthopaedics, VMKV Medical College and Hospital, Salem, Tamilnadu, India. ${ }^{4}$ Department of Physiotherapy, VMKV Medical College and Hospital, Salem, Tamilnadu, India.

\section{ABSTRACT}

\section{BACKGROUND}

Total Knee Arthroplasty (TKA) has become a standard operative procedure to relieve pain, and to restore alignment and function of patients with advanced arthritis of knees.1,2,3 Total knee arthroplasty has evolved into a very reliable surgical treatment for advanced arthritis of knee. TKA provides pain relief and improves function for patients with advanced osteoarthritis of the knee. The aim of this study is to prospectively evaluate the clinical and functional outcomes of total knee arthroplasty in arthritic knees in terms of pain relief, range of motion and stability.

\section{METHODS}

We prospectively reviewed a series of 65 primary Total Knee Arthroplasties performed in 40 patients at our institute from November 2014 to November 2017. Of these patients, 12 underwent bilateral TKA in the same sitting, 13 underwent staged bilateral TKA and 15 patients underwent TKA on one side. Patients were followed up to 3 years post-operatively with an average follow up of 24 months and were evaluated with Knee Society Clinical Ratings and radiographic analysis.

\section{RESULTS}

Pre-operative mean of clinical score (pain, ROM and stability) and functional score (walking distance and stair climbing) of patients improved from 48.33 to 92.82 and 49.65 to 88.43 respectively.

\section{CONCLUSIONS}

In this short-term analysis of this prospective observational study, satisfactory results were obtained in a good percentage of arthritic knee patients.
Corresponding Author: Dr. Meenakshi Sundaram Kandasamy, VMKV Medical College and Hospital, NH 47, Sankari Main Road, Salem-636308, Tamilnadu, India. E-mail: meenakshiortho@yahoo.com

DOI: $10.14260 /$ jemds/2020/31

Financial or Other Competing Interests: None.

How to Cite This Article:

Kandasamy MS, Kandaswamy KGS, Rajaram SK, et al. Cemented total knee arthroplasty- a short term clinical, functional assessment and outcome analysis. J. Evolution Med. Dent. Sci. 2020;9(03):138-141, 10.14260/jemds/2020/31

Submission 18-10-2019, Peer Review 02-01-2020, Acceptance 08-01-2020, Published 20-01-2020.

\section{KEY WORDS}

TKA, Degenerative Arthritis, Knee Joint, KSCRS 


\section{BACKGROUND}

Total Knee Arthroplasty (TKA) has become a standard operative procedure to relieve pain, and restore alignment and function of patients with advanced arthritis of knees. ${ }^{1,2,3}$ Total knee arthroplasty has evolved into a very reliable surgical treatment for advanced arthritis of knee. TKA provides pain relief and improves function for patients with advanced osteoarthritis of the knee.

As the world population ages and changes in lifestyle produce more degeneration over the weight-bearing joints, more men and women suffer from advanced osteoarthritis and its associated pain and disability. Increasing number of adults with knee arthritis choose TKA to relieve pain and improve function. TKA provides pain relief and improves function for patients with advanced osteoarthritis of the knee 4 . Several studies have been conducted on the outcome of TKA and the results have been steadily improving 5 .

The functional results and longevity of present day TKA using advanced prosthetic designs, instrumentation and operative techniques are excellent when compared to the TKA utilizing early prosthetic designs. Most of the prosthetic designs are variations of original condylar design. TKA has become a standard treatment of choice and the indications for this procedure have become much more liberal. ${ }^{6}$

\section{METHODS}

We prospectively reviewed the outcomes of a series of TKA surgeries to examine the effectiveness of this procedure in relieving pain and restoring function in knees. The study was conducted in Vinayaka Mission Hospital, Salem between November 2014 to November 2017 and 65 primary TKAs were performed in 40 patients and the outcomes were evaluated prospectively. We included patients with severe degenerative changes aged more than 45 years willing for surgery and excluded the bedridden and severely obese patients.

The mean age of the patients was 62.5 years $(47-78$ years). There were 32 female and 8 male patients. All the patients who underwent surgery here were diagnosed to have advanced degenerative arthritis of knee (36- Osteoarthritis and 4- Rheumatoid Arthritis). 25 patients had bilateral TKA out of which 12 patients underwent surgery at the same sitting indications being bilateral severe angular deformity and bilateral severe flexion contracture ${ }^{7}$. In 13 patients the TKA was performed as staged procedure whereas in 12 patients it was done within duration of 5-8 days and in one patient after a year. 15 patients had unilateral TKA. The decision for staged versus simultaneous procedure was also made on the basis of patients request and financial considerations. Before surgery all patients were subjected to a comprehensive medical evaluation.

The patients were evaluated clinically and radiographically before operation, immediate postoperatively, at discharge, 1 month and 1 year post-operatively and yearly thereafter. Antero-posterior and lateral radiographs in weight bearing position were made routinely. Radiological grading system as advocated by Kellegren and Lawrence ${ }^{2,8}$ was used pre-operatively to evaluate the severity of arthritis. These same radiographs were obtained at subsequent post-operative visits to recalculate alignment and the position of components. The overall alignment of extremity, as well as the orientation of the femoral and tibial components to the respective bones was documented at each radiographic evaluation. Varus to valgus deformity ratio was 33:7 and preoperative planning to correct the deformity was done. Average pre-operative varus deformity and valgus deformity were 15 degrees (range -8 to 20 degrees) and 10 degrees (range -5 to 14 degrees) respectively.

All the procedures were performed using same pre, intra and post-operative protocols. The procedure was performed under the control of tourniquet. A standard anterior midline incision on skin followed by a medial parapatellar arthrotomy was used to expose the knee in all the patients. Adequate soft tissue release was performed to correct varus, valgus and flexion contracture. Routinely in all cases patellar replacement was not done and only patellar denervation with nibbling of osteophytes was done. Bone cuts were made appropriately using the standard alignment method (extra-medullary alignment for tibia and intramedullary for femur). Precision jigs/guides were used to help make sure the cuts are made at the correct angles so the bones would align properly after the implants are fixed. The fixation of components was done by cementing. Wound was closed in layers with suction drain insitu and compression bandage applied. Selected patients who were having excruciating pain were put on long knee brace in early post-operative period to assist with walking and to help minimize pain.

Total blood loss both intra-operative and post-operative suction drain was around $300 \mathrm{ml}$ on an average per knee. All the patients were attended by Physiotherapist pre-operatively and post-operative protocols were instructed. They were advised to perform isometric quadriceps exercises and assisted SLRs on the first post-operative day. Knee flexion in limited/ controlled range was started from second postoperative day onwards and progressed daily. CPM $^{9}$ was instituted in few patients in whom the knee movements were not satisfactory. Knee flexion was delayed in cases of questionable viability of wound margins. Ambulation with walker was started on individual basis based on the muscle power and patient's motivation from $3^{\text {rd }}$ to $4^{\text {th }}$ post-operative day and progressed gradually to single elbow crutch which the patients used till $4^{\text {th }}$ week. All the patients had DVT prophylaxis until the satisfactory ambulation status was achieved and they were discharged during the $2^{\text {nd }}$ postoperative week after the suture removal.

The patients were followed up at $4^{\text {th }}$ week, $12^{\text {th }}$ week, 6 months, and 1 year and then yearly. Patients were assessed using clinical scoring by Knee Society Clinical Rating System,10,11,12,3 functional scoring and X-rays to evaluate alignment and implant position.

\section{RESULTS}

At an average of 24 months postoperatively, patients were assessed using KSCRS index to rate their pre-operative and present joint pain, stiffness and function. In patients who turned up for the follow-up examinations, the under listed are the variables which were studied and analysed. 
1. Age- Average of 62.5 years (range 47 to 78 years).

2. Sex- Females - 32, Males - 8 (we observed no notable gender difference in the pre- and post-operative functional outcomes).

3. Side- Unilateral in 15 patients. Bilateral in 25 patients ( 12 of bilateral patients underwent surgery at the same sitting).

4. Diagnosis- Osteoarthritis knee in 36 patients and Rheumatoid Arthritis in 4 patients.

5. Severity of Arthritis- 74\% ( $n=48)$ knees had Grade IV arthritis (Kellegren and Lawrence scoring system).

6. Number of Patients- Who have not completed at least 1 year of follow-up: 5 (12.5\%)

7. Implants- Imported: 65, Indian: 0, PFC sigma: 52, Hiflexion/ RPF: 13.

8. Clinical \& Functional Outcome- Both clinical and functional scores (KSCRS. ${ }^{10,11,12,3}$ ) (Table 1) calculated with each mounting to a total of 100 points.

\section{Clinical Score}

Total 100 points [Pain -50 points + ROM -25 points + Stability -25 points]

\section{Functional Score}

Total 100 points [Walking distance -50 points + Stair climbing -50 points]

At the time of latest follow-up, pain was adequately relieved in 54 knees (83.08\%) and the average range of flexion was 120 degrees (range 108 to 127 degrees) and all the knees $(100 \%)$ had normal anteroposterior and mediolateral stability both clinically and radiographically.

\begin{tabular}{|c|c|}
\hline Grade - Score & No. of Knees (\%) \\
\hline Excellent $(>85)$ & $24(36.92)$ \\
\hline Good $(70-84)$ & $28(43.08)$ \\
\hline Fair $(60-69)$ & $10(15.38)$ \\
\hline Poor $(<60)$ & $3(4.61)$ \\
\hline \multicolumn{2}{|c|}{ Table 1. Grading of Results Based on KSCRS in This Study } \\
\hline
\end{tabular}

\section{DISCUSSION}

We prospectively reviewed 40 consecutive patients (65 TKAs) with advanced degenerative arthritis who had undergone primary TKA between November 2014 and November 2017. All subjects were implanted with prosthesis using bone cement fixation for all components. The femoral components were identical, and the tibial component type was either mobile bearing rotating platform tibial design or a fixed bearing tibial design. The selection of a fixed vs. mobile bearing TKA was primarily based on age and activity level of patients.

Pre-operative mean of clinical score (pain, ROM and stability) and functional score (walking distance and stair climbing) of patients improved from 48.33 to 92.82 and 49.65 to 88.43 respectively. All the cases were performed with soft tissue balancing except in 2 patients in whom valgus deformity was present a planned lateral femoral condyle sliding osteotomy followed by cancellous screw fixation prior to cementing and implant fixation was performed to achieve the mechanical alignment. Those knees were immobilized in long knee brace for one month. Seven patients reported minimal knee pain at the 4-week post-operative follow-up, which improved by exercises and analgesics. All patients except one reported complete relief of pain after the procedure and none required analgesics until their last follow-up visits. None of the patients had post-operative peri-prosthetic fracture. Due to the short duration of the study, long-term complications like implant failure, loosening and osteolysis were not encountered.

One patient had delayed wound healing due to superficial infection which improved with basic wound care and systemic antibiotics. She had no evidence of infection at any of the subsequent follow-up visits. Total knee arthroplasty has been shown to be effective in decreasing pain and restoring function and alignment in arthritic knees ${ }^{13}$. Common to most of the reports is a classification of success or failure based on the clinical and radiographic criteria of the involved knee and the physician's perception of the patient's ability to perform basic functions of walking and stair-climbing. ${ }^{14}$

Another common outcome indicator for success is the implant survival. Given the increasingly good functional results with modern arthroplasty techniques, implant survival in TKA patients is becoming a less critical issue in the more elderly patient ${ }^{15}$. There is now a national emphasis on expanding evaluation of outcomes to include self-reported measures of functional assessment, including pain and mobility, but also social and global factors of overall quality of life. ${ }^{16}$ The goal of this study was to describe the patientassessed outcomes and to evaluate predictors of patient satisfaction following TKA. About 52 of our patients had good to excellent grading of functional outcome.

The results of our study showed significant improvement in pain and walking ability following TKA. The self-reported functional status scores appeared to be good predictors of patient satisfaction in our study. We found no correlation between patient satisfaction and age, sex, weight, or any other preoperative medical comorbidities. It appears that the patient satisfaction is more closely associated with patientassessed quality of life variables. ${ }^{17}$

Achieving painless, stable, deformity free, mobile joint is the ultimate aim of TKA. Thorough clinical and radiological evaluation, strict patient selection, improvised design, improved surgical technique, an experienced team and a wellestablished centre are the pre-requisites of achieving uncompromising results.

\section{CONCLUSIONS}

TKA is vastly useful procedure for degenerative arthritis of the knee with a high level of functional outcome. The TKA prosthesis has been well accepted by our patients, both in terms of overall functional ability in general and knee function in particular. The type of implants did not influence the ultimate outcome of the results in a statistically significant manner. While this study has some limitations, we believe none are likely to alter the conclusion. Careful preoperative planning and precise surgical technique help in avoiding unexpected intra-operative difficulties and achieve a successful outcome. 


\section{REFERENCES}

[1] Yang CC, McFadden LA, Dennis DA, et al. Lateral retinacular release in mobile-versus fixed-bearing TKA. Clin Orthop Relat Res 2008;466(11):2656-61.

[2] Emrani PS, Katz JN, Kessler CL, et al. Joint space narrowing and Kellgren-Lawrence progression in knee osteoarthritis: an analytic literature synthesis. Osteoarthritis Cartilage 2008;16(8):873-82.

[3] Kiran KE, Malhotra R, Bhan S. Unilateral vs. one stage bilateral total knee replacement in rheumatoid and osteoarthritis - a comparative study. Indian Journal of Orthopaedics 2005;39(1):14-20.

[4] Franklin PD, Li W, Ayers DC. The Chitranjan Ranawat Award: functional outcome after total knee replacement varies with patient attributes. Clin Orthop Relat Res 2008;466(11):2597-604.

[5] Woolhead GM, Donovan JL, Dieppe PA. Outcomes of total knee replacement: a qualitative study. Rheumatology (Oxford) 2005;44(8):1032-7.

[6] Kemparaju N, Kumaresan P, Jayasankar. Total knee arthroplasty in arthritic knee: our experience. Journal of Tamil Nadu Orthopaedic Association 2001;27:60-4.

[7] Dhaon BK, Sharma V, Jaiswal A. Simultaneous bilateral versus unilateral total knee replacement in osteoarthritic knee. Ind J of Orthop 2002;36(4):234-7.

[8] Mohan G, Arunmozhirajan M, Sankaralingam K. Functional analysis of cruciate retaining vs cruciate sacrificing prosthesis in total knee replacement. Journal of Tamil Nadu Orthopaedic Association 2009;35:54-8.
[9] Brotzman SB. Clinical orthopaedic rehabilitation. Mosby Year Book Inc., 1996: p. 302-10.

[10] Canale ST. Campbell's Operative Orthopaedics. Vol. 1. $9^{\text {th }}$ edn. Mosby - Year Book Inc., 1998: p. 251-2.

[11] Liow RY, Walker K, Wajid MA, et al. The reliability of the American Knee Society Score. Acta Orthop Scand 2000;71(6):603-8.

[12] Mohan G, Vijayaraghavan PV, Kumar N. Analysis of functional and clinical outcome of total knee arthroplassty in arthritis. Journal of Tamil Nadu Orthopaedic Association 2005;31:56-61.

[13] Laskin RS. The classic: total condylar knee replacement in patients who have rheumatoid arthritis: a ten-year follow-up study. Clin Orthop Relat Res 2008;466(11):2589-96.

[14] Insall JN, Dorr LD, Scott RD, et al. Rationale of The Knee Society clinical rating system. Clin Orthop Relat Res 1989;(248):13-4.

[15] McAuley JP, Collier MB, Hamilton WG, et al. Posterior cruciate-retaining total knee arthroplasty for valgus osteoarthritis. Clin Orthop Relat Res 2008;466(11):26449.

[16] Freund DA, Dittus RS, Fitzgerald J, et al. Assessing and improving outcomes: total knee replacement. Health Serv Res 1990;25(5):723-6.

[17] Anderson JG, Wixson RL, Tsai D, et al. Functional outcome and patient satisfaction in total knee patients over the age of 75. The Journal of Arthroplasty 1996;11(7):831-40. 